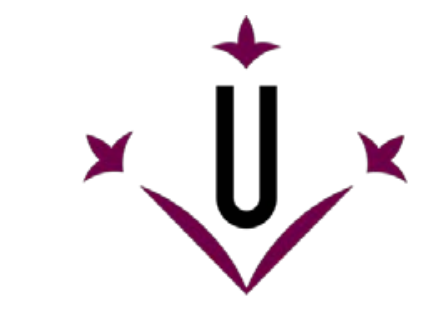

Universitat de Lleida

Document downloaded from:

http://hdl.handle.net/10459.1/66460

The final publication is available at:

https://doi.org/10.1007/s00009-013-0360-3

Copyright

(c) Springer Basel, 2014 


\title{
THE JUMPING KNIGHT AND OTHER (SUPER) EDGE-MAGIC CONSTRUCTIONS
}

\author{
S.C. LÓPEZ, F.A. MUNTANER-BATLE, AND M. RIUS-FONT
}

\begin{abstract}
Let $G$ be a graph of order $p$ and size $q$ with loops allowed. A bijective function $f: V(G) \cup E(G) \rightarrow\{i\}_{i=1}^{p+q}$ is an edge-magic labeling of $G$ if the sum $f(u)+f(u v)+f(v)=k$ is independent of the choice of the edge $u v$. The constant $k$ is called either the valence, the magic weight or the magic sum of the labeling $f$. If a graph admits an edge-magic labeling, then it is called an edge-magic graph. Furthermore, if the function $f$ meets the extra condition that $f(V(G))=\{i\}_{i=1}^{p}$ then $f$ is called a super edge-magic labeling and $G$ is called a super edge-magic graph. A digraph $D$ admits a labeling, namely $l$, if its underlying graph, und $(D)$ admits $l$.

In this paper, we introduce a new construction of super edge-magic labelings which is related to the classical jump of the knight on the chess game. We also use super edge-magic labelings of digraphs together with a generalization of the Kronecker product in order to get edge-magic labelings of some families of graphs.
\end{abstract}

\section{INTRODUCTION}

For the undefined concepts and notation used in this paper, we refer the reader to either one the following sources $[4,5,14,20]$. However, in order to make this paper reasonably self contained, we mention that by a $(p, q)$-graph we mean a graph of order $p$ and size $q$. We also point out that the graphs and digraphs used in this paper may contain loops. Whenever we refer to graphs without loops we will call them simple graphs. Let $C_{n}$ be the cycle of order $n$. We denote by $C_{n}^{+}$and by $C_{n}^{-}$the two possible strong orientations of the cycle $C_{n}$ and we use the expression $\vec{G}$ to denote an oriented graph obtained from a graph $G$. We also denote by $C_{1}$ a loopgraph, that is a graph of order 1 and size 1. A digon formed by two different vertices $x$ and $y$ of a digraph is a direct cycle with set of $\operatorname{arcs}\{(x, y),(y, x)\}$.

Kotzig and Rosa [15] introduced in 1970 the concept of edge-magic labelings of simple graphs. Let $G$ be a simple $(p, q)$-graph and let $f: V(G) \cup E(G) \longrightarrow\{i\}_{i=1}^{p+q}$ be a bijective function such that the sum $f(u)+f(u v)+f(v)=k$ for any edge $u v \in E(G)$. Then $f$ is an edge-magic labeling of $G, G$ is called an edge-magic simple graph and $k$ is called either the valence, the magic weight or the magic sum of $f$ (see $[4,14,20])$.

Later on, motivated by the concept of 'edge-magicness', Enomoto, Lladó, Nakamigawa and Ringel [6], defined in 1998 the concept of super edge-magic labelings of simple graphs. Let $G$ be a simple $(p, q)$-graph. A super edge-magic labeling of $G$ is

Date: December 3, 2012.

1991 Mathematics Subject Classification. Primary 05C78.

Key words and phrases. (super) edge-magic, Jacobsthal sequence, dual shuffle prime, $\otimes_{h^{-}}$ product. 
an edge-magic labeling $f$ of $G$ with the extra property that $f(V(G))=\{i\}_{i=1}^{p}$. In this case, $G$ is said to be a super edge-magic simple graph. An interesting family of super edge-magic simple graphs that will be of great use in the rest of this paper is the family of cycles of odd order. In fact, a cycle is super edge-magic if and only if its order is odd. It is worth to mention that the concept of super edge-magic graphs was introduced by Enomoto et al. and these authors were unaware of the fact that Acharya and Hegde had introduced in [1] strongly indexable graphs. Although the motivations for introducing these two concepts were different, it is easy to check that the two concepts are equivalent.

The concepts of edge-magic labelings and super edge-magic labelings were generalized to graphs in general [11] in the obvious way. That is to say, the valence of a loop $u u$ equals to $2 f(u)+f(u u)$.

It was also in [11] that the concept of (super) edge-magic labeling of a digraph $D$ was introduced as follows: a digraph $D$ is (super) edge-magic if its underlying graph, und $(D)$, is (super) edge-magic. In general, a digraph is said to admit a labeling $l$ if its underlying graph admits $l$.

The following characterization of super edge-magic simple graphs was introduced in [7]:

Lemma 1.1. Let $G$ be a simple $(p, q)$-graph. Then $G$ is super edge-magic if and only if there is a bijective function $g: V(G) \longrightarrow\{i\}_{i=1}^{p}$ such that the set $S=$ $\{g(u)+g(v): u v \in E(G)\}$ is a set of $q$ consecutive integers. In this case, $g$ can be extended to a super edge-magic labeling $f$ of $G$.

Although the characterization was introduced for simple graphs it is the case that Lemma 1.1 works for graphs as well, and this generalization is so useful that, unless otherwise specified, whenever we refer to a super edge-magic labeling of a graph, from now on, we will refer to a function as the function $g$ described in the statement of Lemma 1.1.

1.1. Dual shuffle primes. Mathematicians have been studying permutations for many years. Let $n \in \mathbb{N}$. A permutation $\pi$ is a bijective mapping $\pi:\{i\}_{i=1}^{n} \rightarrow\{i\}_{i=1}^{n}$. It is well known that a common way to describe permutations is by means of the union of mutually disjoint strongly oriented cycles, in which $(i, j)$ is an arc of an oriented cycle if and only if $\pi(i)=j$. We will refer to this representation simply as the cycle representation of $\pi$.

Among permutations, the ones arising from perfectly shuffling a deck of cards have received special attention. Let us define our deck of cards in such a way that each card receives a number from 1 up to $n$, the number of cards in the deck, the card numbered 1 lying at the bottom of the deck, the card numbered 2 lying on top of the card numbered 1 and so on until we reach the card numbered $n$. We will call this ordering of the deck $\alpha_{n}$ and this will be our initial ordering (the standard word in terms of [3]).

Next we will describe three possible ways to perfectly shuffle $\alpha_{n}$, and we will denote, following Asveld's notation in [3], these three ways by $S_{\bullet}, S$ and $\bar{S}$. The first perfect shuffle operation, $S_{\bullet}$, called the original perfect shuffle operation, consists on cutting a deck of an even number of cards $n$ into two equal parts, and then interleaving these two parts. Hence, applying this operation to the initial ordering $\alpha_{n}, n$ even, we obtain: $S_{\bullet}\left(\alpha_{n}\right)=1, k, 2, k+1,3, k+2, \ldots$, where $k=\lceil(n+1) / 2\rceil$. It is clear that $S_{\bullet}\left(\alpha_{n}\right)$ can be obtained applying a permutation $\pi\left(S_{\bullet}\right)$ to $\alpha_{n}$, however 
$\pi\left(S_{\bullet}\right)$ always fixes 1 . In order to avoid this fixed point, we can slightly modify the operation $S_{\bullet}$, by changing the way in which we are interleaving the cards. We apply the operation $S$ on $\alpha_{n}$ such that the resulting order is, when $n$ is even $S\left(\alpha_{n}\right)=k, 1, k+1,2, k+2,3, \ldots$, where $k=\lceil(n+1) / 2\rceil$. When $n$ is odd, we isolate $n$ and put it on top of the shuffled deck. Once again, $S\left(\alpha_{n}\right)$ can be obtained by applying a permutation $\pi(S)$ on $\alpha_{n}$, and in this case, it is not obvious to decide for which even $n, \pi(S)$ has fixed points. Furthermore, it is not clear when the cycle representation of $\pi(S)$ consists of a unique oriented cycle, or it is the vertex disjoint union of oriented cycles.

An operation on $\alpha_{n}$, that we will pay special attention to, is $\bar{S}$. This reordering operation, in a way, can be thought as the dual of $S$, and hence, it is known as the dual shuffle operation:

$$
\bar{S}\left(\alpha_{n}\right)= \begin{cases}k-1, n-1, k-2, n-2, \ldots, 1, k, n, & \text { when } n \text { odd, } \\ k-1, n, k-2, n-1, \ldots, 1, k, & \text { when } n \text { even, }\end{cases}
$$

where $k=\lceil(n+1) / 2\rceil$. In this case, it is clear that $\pi(\bar{S})$ can be defined according to the parity of $n$ as follows: if $n$ is even then $\pi(\bar{S})(m) \equiv-2 m(\bmod n+1)$, and if $n$ is odd, then $\pi(\bar{S})(m) \equiv-2 m(\bmod n)$, for $1 \leq m<n$, and $\pi(S)(n)=n$. When the cycle representation associated to the dual shuffle operation on $\alpha_{n}$ consists of a unique cycle, we say that $n$ is a dual shuffle prime. An interesting question is to find an explicit formula for the $n^{\text {th }}$ - dual shuffle prime. We list below the first elements of the set of dual shuffle primes, namely DSP. A more complete list can be found in [19].

$$
\mathrm{DSP}=\{4,6,12,22,28,36,46,52, \ldots\} .
$$

Super edge-magic labelings are of importance among graph labelings due to the great amount of relations that they have with other labelings (see $[7,11,13,16,17])$. In particular, super edge-magic labelings of 2-regular graphs have proven to be of the great help in order to find links among labelings. Motivated by this fact, one of the goals of this paper is to use operations on $\alpha_{n}$ in order to be able to find super edge-magic labelings of 2-regular graphs and other related graphs.

1.2. Two special partitions. In this paper, two special partions of the set $\{1,2, \ldots, n\}$ emerge when studying the graphs obtained by our constructions. One type is of the form $\cup_{k=1}^{n} \Theta_{k}$, when $n$ is odd, and the other one is of the form $\cup_{k=1}^{n} \Phi_{k}$, when $n$ is even. Those sets, $\Theta_{k}$ and $\Phi_{k}$ are introduced just before Theorem 2.9 and Theorem 3.7 , respectively, in a pure arithmetic manner.

1.3. Digraph products applied to labelings. Figueroa-Centeno et al. introduced the following product of digraphs in [11]: let $D$ be a digraph and let $\Gamma=$ $\left\{F_{i}\right\}_{i=1}^{m}$ be a family of digraphs such that $V\left(F_{i}\right)=V$ for every $i \in\{j\}_{j=1}^{m}$. Consider any function $h: E(D) \longrightarrow \Gamma$. Then the product $D \otimes_{h} \Gamma$ is the digraph with vertex set $V(D) \times V$ and $((a, b),(c, d)) \in E\left(D \otimes_{h} \Gamma\right)$ if and only if $(a, c) \in E(D)$ and $(b, d) \in E(h(a, c))$. The adjacency matrix of $D \otimes_{h} \Gamma, A\left(D \otimes_{h} \Gamma\right)$, is obtained by multiplying every 0 entry of $A(D)$, the adjacency matrix of $D$, by the $|V| \times|V|$ null matrix and every 1 entry of $A(D)$ by $A(h(a, c))$, where $(a, c)$ is the arc related to the 1 entry of $A(D)$. Notice that, when $h$ is constant, $A\left(D \otimes_{h} \Gamma\right)$ coincides with the classical Kronecker product of matrices, $A(D) \otimes A(h(a, c))$. When $|\Gamma|=1$, we just write $D \otimes \Gamma$. 
Let $\mathcal{S}_{n}$ denote the set of all 1-regular super edge-magic labeled digraphs of order $n$ where each vertex takes the name of the label assigned to it. The following results were also introduced in [11]:

Theorem 1.2. [11] Assume that $D$ is any (super) edge-magic digraph and let $h: E(D) \longrightarrow \mathcal{S}_{n}$ be any function. Then und $\left(D \otimes_{h} \mathcal{S}_{n}\right)$ is (super) edge-magic.

Theorem 1.3. [11] Let $h$ be a function that assignes to each of the arcs of the digraph $C_{m}^{+}$the same strong orientation of the cycle $C_{n}$. Then und $\left(C_{m}^{+} \otimes_{h} \mathcal{S}_{n}\right) \cong$ $\operatorname{gcd}(m, n) C_{l c m(m, n)}$.

Theorem 1.2 has been extended by replacing the set $\mathcal{S}_{n}$ by the set $\mathcal{S}_{n}^{k}$ in [17]. A super edge-magic labeled digraph $F$ is in $\mathcal{S}_{n}^{k}$ if $|V(F)|=|E(F)|=n$ and the minimum sum of the labels of the adjacent vertices is equal to $k$. An easy computation shows that $\mathcal{S}_{n} \subset \mathcal{S}_{n}^{(n+3) / 2}$ and therefore, the next result is a generalization of Theorem 1.2.

Theorem 1.4. [17] Assume that $D$ is any (super) edge-magic digraph and let $h: E(D) \longrightarrow \mathcal{S}_{n}^{k}$ be any function. Then und $\left(D \otimes_{h} \mathcal{S}_{n}^{k}\right)$ is (super) edge-magic.

As the title of the paper indicates, we will devote these pages to construct edgemagic labelings of some infinite families of graphs. One of the main tools will be the $\otimes_{h}$-product. In what follows we introduce the necessary notation and terminology as well as a brief survey of what is known about these families.

Let $G$ and $H$ be two (di)graphs and let $u$ be a distinguished vertex of $G$. Let $H * u G$ be the (di)graph obtained from $H$ and $G$ by gluing a copy of $G$ to each vertex $x$ of $H$, by means of identifying $x$ to the vertex related to $u$ in the given copy. Note that, in many circumstances, the resulting (di)graph $H * u G$ is independent of the choice of the vertex $u$ in $G$. In sections 4 and 5, we concentrate our attention on the cases when $H$ and $G$ are both cycles and when $H$ is a cycle and $G$ is a path.

Next, we introduce the following related results.

Theorem 1.5. [17] Let $m$ and $n$ be positive odd integers with $m \equiv 3$ ( $\bmod 4)$ and $n \geq 3$. If $m \geq n$ then $C_{n} * u C_{m}$ is super edge-magic, $u \in V\left(C_{m}\right)$.

Let $P_{2 n+1}$ denote the path of order $2 n+1$. We have the following result.

Theorem 1.6. [17] If $C_{m}$ is (super) edge-magic and $v$ is the central vertex of $P_{2 n+1}$, then $C_{m} * v P_{2 n+1}$ is (super) edge-magic.

The following open question can be found in [17].

Question 1.1. [17] Characterize the pairs $(n, m)$ for which $C_{n} * u C_{m}, u \in V\left(C_{m}\right)$, is super edge-magic.

In this paper we introduce the following open question and we devote section 4 to throw some light towards a possible solution.

Question 1.2. Characterize the pairs $(n, m)$ for which $C_{n} * u C_{m}, u \in V\left(C_{m}\right)$, is edge-magic.

The next results can be found in [11] and [2].

Theorem 1.7. [11] Let $G$ be any bipartite graph with stable sets $V_{1}$ and $V_{2}$ and let $\vec{G}$ be the digraph obtained from $G$ by orienting each edge of $E(G)$ in such a way that if $(a, b) \in E(\vec{G})$ then $a \in V_{1}$ and $b \in V_{2}$. Then und $\left(\vec{G} \otimes C_{n}^{+}\right) \cong n G$. 
Theorem 1.8. [11] Let $\vec{T}$ be any orientation of a tree $T$, and let $h: E(\vec{T}) \rightarrow \mathcal{S}_{n}$ be any function. Then und $\left(\vec{T} \otimes_{h} \mathcal{S}_{n}\right) \cong n T$.

Theorem 1.9. [2] Let $m, n \in \mathbb{N}$ and consider the product $C_{m}^{+} \otimes_{h}\left\{C_{n}^{+}, C_{n}^{-}\right\}$where $h: E\left(C_{m}^{+}\right) \longrightarrow\left\{C_{n}^{+}, C_{n}^{-}\right\}$. Let $g$ be a generator of a cyclic subgroup of $\mathbb{Z}_{n}$, namely $\langle g\rangle$, such that $|\langle g\rangle|=k$. Also let $N_{g}\left(h^{-}\right)<m$ be a positive integer that satisfies the following congruence relation $m-2 N_{g}\left(h^{-}\right) \equiv g(\bmod n)$.

If the function $h$ assigns $C_{n}^{-}$to exactly $N_{g}\left(h^{-}\right)$arcs of $C_{m}^{+}$, then the product $C_{m}^{+} \otimes_{h}\left\{C_{n}^{+}, C_{n}^{-}\right\}$consists of exactly $n / k$ disjoint copies of a strongly oriented cycle $C_{m k}^{+}$. In particular if $g c d(g, n)=1$, then $\langle g\rangle=\mathbb{Z}_{n}$ and if the function $h$ assigns $C_{n}^{-}$ to exactly $N_{g}\left(h^{-}\right)$arcs of $C_{m}^{+}$then

$$
C_{m}^{+} \otimes_{h}\left\{C_{n}^{+}, C_{n}^{-}\right\} \cong C_{m k}^{+} .
$$

The organization of the paper is the following one. We construct super edgemagic labelings of 2-regular graphs using the classical jump of the knight on the chess game in section 2. With a slight modification we obtain super edge-magic labelings of the union of 2-regular graphs with a $K_{2}$ in section 3 . In sections 4 and 5 we study the edge-magicness of $C_{n} * u C_{m}$ and $C_{n} * u P_{m}$, respectively, using the $\otimes_{h}$-product of digraphs. Some further results obtained from the previous sections are given in section 6. A more detailed summary of the results presented in the paper is given in the final subsection 6.1.

\section{Super EDGe-MAgic LABELINGS OF 2-Regular GRAPHS}

It is well known that if a $(p, q)$-graph $G$ is super edge-magic then $q \leq 2 p-3$ (see [6]). If $G$ is bipartite, then this bound can be improved to $q \leq 2 p-5$ (see [9]). These bounds imply that if an $r$-regular graph is super edge-magic, then $r \in\{0,1,2,3\}$. It is clear that 0-regular graphs are of no interest in this context. Furthermore, 1-regular super edge-magic graphs have been completely characterized in [15] by Kotzig and Rosa. Therefore, it seems to be a natural question to ask which 2-regular graphs are super edge-magic. This is what was in the minds of Figueroa-Centeno et al. in [10] when they conjectured that the graph $C_{m} \cup C_{n}$ is super edge-magic if and only if $m+n$ is odd and greater than 1 . Holden et al. went further into this conjecture, although they arrived to it from a different point of view, when they conjectured in [12] that all 2-regular graphs of odd order are strong vertex total magic, excluding $C_{3} \cup C_{4}, 3 C_{3} \cup C_{4}$ and $2 C_{3} \cup C_{5}$, which is in fact equivalent to saying that they are super edge-magic.

In this section, we establish a new relationship between super edge-magic labelings of 2-regular graphs and $\pi(\bar{S})$. Let $D_{\lambda}$ be a super edge-magic digraph $D$ with a super edge-magic labeling $\lambda$, such that each vertex of $D$ takes the name of its label under $\lambda$. Then, the adjacency matrix of $\left.D_{\lambda}, A\left(D_{\lambda}\right)\right)=\left(a_{i j}\right)$, is defined as follows: if $(i, j) \in E\left(D_{\lambda}\right)$ then $a_{i j}=1$, otherwise $a_{i j}=0$. From Lemma 1.1, it is clear that, if we let the diagonals from left to right and bottom to up be the counterdiagonals, then the set of counterdiagonals containing a 1 is a consecutive set. Furthermore, each counterdiagonal contains at most a 1 . If $D_{\lambda}$ is a digraph obtained from a 2-regular graph by attaching a cyclic orientation to each component, that is to say, $D_{\lambda}$ is a 1-regular digraph, then $A\left(D_{\lambda}\right)$ has the extra property that each column and each row contains exactly a 1 . Moreover, it is clear that if a square matrix $A$ of 0 's and 1's meets the following properties: (i) each counterdiagonal contains at most one 1 , (ii) the counterdiagonals containing exactly a 1 form a set of consecutive 
counterdiagonals and, (iii) each row and each column of the matrix have all entries being 0 , except for exactly one entry which is 1 , then $A$ is the adjacency matrix of some super edge-magic labeled 1-regular digraph.

2.1. The jumping knight. Let $A_{n}=\left(a_{i j}\right)$ be the square matrix of dimension $n$ defined by $a_{i j}=1$ if $j \equiv-2 i+2(\bmod n)$ and $a_{i j}=0$, otherwise. Similarly, let $A_{n}^{\prime}=\left(a_{i j}^{\prime}\right)$ be the square matrix of dimension $n$ defined by $a_{i j}^{\prime}=1$ if $j \equiv-2 i+1$ $(\bmod n)$ and $a_{i j}^{\prime}=0$, otherwise. Observe that the positions of the 1 entries of the matrices $A_{n}$ and $A_{n}^{\prime}$ so defined, correspond to the jump of the knight on the $n \times n$ chessboard, when the matrices are viewed as chessboards with exactly $n^{2}$ squares.

Let $G_{n}$ and $G_{n}^{\prime}$ be the digraphs with vertex sets $V\left(G_{n}\right)=V\left(G_{n}^{\prime}\right)=\{1,2, \ldots, n\}$ and adjacency matrices $A_{n}$ and $A_{n}^{\prime}$, respectively. The function $f(x)=n+1-x$ from $V\left(G_{n}\right)$ onto $V\left(G_{n}^{\prime}\right)$ defines an isomorphism of the digraphs $G_{n}$ and $G_{n}^{\prime}$. Clearly, there is an $\operatorname{arc}(i, j) \in E\left(G_{n}\right)$ if and only if, $j \equiv-2 i+2(\bmod n)$. Similarly, there is an arc $\left(n+1-i, j^{\prime}\right) \in E\left(G_{n}^{\prime}\right)$ if and only if, $j^{\prime} \equiv-2(n+1-i)+1(\bmod n)$, that is, if and only if, $j^{\prime}=n+1-j$.

Lemma 2.1. Let $n$ be an odd integer. Then the digraph $G_{n}$ is a 1-regular super edge-magic digraph.

Proof. It is clear since its adjacency matrix meets the three properties mentioned above.

Lemma 2.2. Let $n$ be an odd integer. The digraph $G_{n}$ contains a cycle of length $k$ if and only if there exists $x \in\{1,2, \ldots, n\}$ such that $k$ is the minimum $i$ with

$$
\left((-2)^{i}-1\right) x \equiv \sum_{j=1}^{i}(-2)^{j}(\bmod n) .
$$

Proof. Let $x \in\{1,2, \ldots, n\}$ and consider the sequence $x_{0}, x_{1}, \ldots, x_{k}$, where $x_{0}=x$ and $x_{j} \equiv-2 x_{j-1}+2(\bmod n)$, for $j=1,2, \ldots, k$. Thus, recursively, we obtain that $x_{j} \equiv(-2)^{j} x-\sum_{l=1}^{j}(-2)^{l}(\bmod n)$. By construction, $x$ is in a cycle of length $k$ if and only if $x_{j} \neq x$, for $j=1,2, \ldots, k-1$ and $x_{k}=x$. Therefore, $x$ is in a cycle of length $k$ if and only if $k$ is the minimum $i$ such that $\left((-2)^{i}-1\right) x \equiv \sum_{l=1}^{i}(-2)^{l}$ $(\bmod n)$.

The next lemma introduces a nice integer sequence that will be used to study the cycle structure of the digraph $G_{n}$.

Lemma 2.3. Let $\left(a_{i}\right)$ be the integer sequence defined by, $a_{1}=a_{2}=1$ and $a_{i}=$ $a_{i-1}+2 a_{i-2}$, for $i \geq 3$. Then

(i) $a_{i}=\left(2^{i}+(-1)^{i+1}\right) / 3$, for each $i \geq 1$.

(ii) $3^{l}$ is a divisor of $a_{3^{l}}$ and $3^{l+1}$ is not.

Proof. Equality (i) is an easy exercise. Since $a_{3}=3$, condition (ii) clearly holds for $l=1$. Suppose that it holds for $1 \leq i \leq l$ and consider the equalities

$$
\frac{a_{3^{l+1}}}{a_{3^{l}}}=\frac{2^{3^{l+1}}+1}{2^{3^{l}}+1}=\left(2^{3^{l}}\right)^{2}-2^{3^{l}}+1=\left(2^{3^{l}}+1\right)^{2}-3 \cdot 2^{3^{l}} .
$$

Thus, we obtain that $a_{3^{l+1}} / a_{3^{l}}=\left(a_{3^{l}}\right)^{2}-3 \cdot 2^{3^{l}}$. Hence, 3 is a divisor of $a_{3^{l+1}} / a_{3^{l}}$ and 9 is not. Therefore, and by the induction hypothesis, we obtain that $3^{l+1}$ is a divisor of $a_{3^{l+1}}$ and $3^{l+2}$ is not. 
By replacing $z$ by -2 in the polynomial factorization $z^{i}-1=(z-1)\left(\sum_{l=0}^{i-1} z^{l}\right)$, for all $i \in \mathbb{N}$, we obtain that

$$
(-2)^{i}-1=-3 \sum_{l=0}^{i-1}(-2)^{l} .
$$

Remark 2.4. The integer sequence introduced in Lemma 2.3 is called the Jacobsthal sequence (or Jacobsthal numbers). This sequence, which appears in [19] as 'A001045', has connections with multiple applications, some of them can be found in [19]. The next result shows another one.

Corollary 2.5. Let $n$ be an odd positive integer and let $\left(a_{i}\right)$ be the Jacobsthal sequence. Then, the digraph $G_{n}$ contains a cycle of length $k$ if and only if there exists $x \in\{1,2, \ldots, n\}$ such that $k$ is the minimum $i$ with $3 a_{i} x \equiv 2 a_{i}(\bmod n)$.

Proof. The result follows from Lemma 2.2, (2.1) and the equality $(-1)^{i} a_{i}=\left((-2)^{i}-\right.$ 1) $/ 3$.

The next lemma characterizes the positive integers $n$ for which the digraph $G_{n}$ contains a cycle of length $3^{l}$.

Lemma 2.6. Let $n$ be an odd integer. Then, $G_{n}$ does not contain digons. Moreover,

(i) If $\operatorname{gcd}(3, n)=1$ then $C_{1}$ is a subgraph of und $\left(G_{n}\right)$.

(ii) If $n=3^{k} n_{1}$ and $\operatorname{gcd}\left(3, n_{1}\right)=1$ then $C_{3^{k}}$ is a subgraph of und $\left(G_{n}\right)$.

(iii) If $n$ is a power of 3 then $\operatorname{und}\left(G_{n}\right) \cong C_{n}$.

Proof. Lemma 2.1 implies that the digraph $G_{n}$ does not contain any digon, since otherwise it is not super edge-magic. Statement (i) clearly holds, since by Corollary 2.5, $C_{1}$ is a subgraph of $\operatorname{und}\left(G_{n}\right)$ if and only if the equation $3 x \equiv 2(\bmod n)$ has a solution. Suppose now that $n=3^{k} n_{1}$ and $\operatorname{gcd}\left(3, n_{1}\right)=1$. Then $C_{3^{s}}$ is not a subgraph of und $\left(G_{n}\right)$, for each $1 \leq s \leq k-1$, otherwise by Corollary 2.5 we have $3 a_{3^{s}} x \equiv 2 a_{3^{s}}(\bmod n)$, for some $x \in\{1,2, \ldots, n\}$ and, by Lemma 2.3 (ii), we would obtain that $3^{s+1} m x \equiv 2 \cdot 3^{s} m(\bmod n)$, where $a_{3^{s}}=3^{s} m$ and $\operatorname{gcd}(3, m)=1$. Thus, by multiplying by $3^{k-1-s} n_{1}$, the congruence $0 \equiv 2 \cdot 3^{k-1} n_{1} m(\bmod n)$ follows, a contradiction. Let us see now that $C_{3^{k}}$ is a subgraph of und $\left(G_{n}\right)$. Let $x$ be a solution of $3 x \equiv 2\left(\bmod n_{1}\right)$. Thus, we obtain that $3^{k+1} m x \equiv 2 \cdot 3^{k} m\left(\bmod 3^{k} n_{1}\right)$, where $a_{3^{k}}=3^{k} \mathrm{~m}$. Hence, by Corollary 2.5, there is a cycle of length $3^{k}$. This proves (ii). Finally, let us see (iii). Let $n=3^{k}$. By using a similar proof as in (ii), it is clear that und $\left(G_{n}\right)$ does not contain a cycle of length $3^{s}$, for $1 \leq s<k$. Moreover, each $x \in\{1,2, \ldots, n\}$ is a solution of the trivial equation $3 a_{3^{k}} x \equiv 2 \cdot a_{3^{k}}$ $(\bmod n)$, since $a_{3^{k}} \equiv 0(\bmod n)$.

Notice that from the previous proof we also conclude that $G_{n}$ contains a cycle of length $3^{k}$, for some integer $k \geq 0$, if and only if $3^{k}$ is a divisor of $n$ and $3^{k+1}$ is not. The characterization of the cycle structure of $G_{n}$ is provided in the next three results.

Proposition 2.7. Let $n$ be an odd prime. Let $k$ be the minimum $i$ such that $(-2)^{i} \equiv 1(\bmod n)$. Then,

$$
\operatorname{und}\left(G_{n}\right) \cong \frac{n-1}{k} C_{k} \cup C_{1} .
$$

Proof. Consider the congruence relation obtained in Corollary 2.5, $3 a_{i} x \equiv 2 a_{i}$ $(\bmod n)$. Hence, since $n$ is prime, either $a_{i} \equiv 0(\bmod n)$, or $3 x \equiv 2(\bmod n)$. 
That is, either $(-2)^{i} \equiv 1(\bmod n)$, or $3 x \equiv 2(\bmod n)$. Therefore, $\operatorname{und}\left(G_{n}\right) \cong$ $(n-1) / k C_{k} \cup C_{1}$, where $C_{1}$ is the graph defined by a loop in the only $x \in\{1,2, \ldots, n\}$ such that $3 x \equiv 2(\bmod n)$.

Corollary 2.8. Let $n+1$ be a prime. Then, $n$ is a dual shuffle prime if and only if, $\operatorname{und}\left(G_{n+1}\right) \cong C_{n} \cup C_{1}$.

Proof. By Theorem 2.11 in [3], $n$ is a dual shuffle prime if and only if, $n+1$ is prime and -2 generates the multiplicative group $\mathbb{Z}_{n+1}^{*}$. Thus, by Proposition 2.7 , this is the case, if and only if, und $\left(G_{n+1}\right) \cong C_{n} \cup C_{1}$.

Let $n$ be an odd integer, $\left(a_{i}\right)$ the Jacobsthal sequence and let $\Theta_{k}$ be a subset of $\{1,2, \ldots, n\}$ defined by $x \in \Theta_{k}$ if $k$ is the minimum $i$ with $3 a_{i} x \equiv 2 a_{i}(\bmod n)$. Then, from Corollary 2.5, it is easy to obtain the following result.

Theorem 2.9. Let $n$ be an odd integer. Then,

$$
\operatorname{und}\left(G_{n}\right) \cong \cup_{k=1}^{n} \frac{\left|\Theta_{k}\right|}{k} C_{k} .
$$

Theorem 2.9 and Lemma 2.1 imply the next result.

Theorem 2.10. Let $n$ be an odd integer. Then, the graph $\cup_{k=1}^{n} \mid\left(\Theta_{k} \mid / k\right) C_{k}$ is super edge-magic.

Notice that, by Theorem 2.9, we immediately obtain that the sets $\Theta_{k}$, for $1 \leq$ $k \leq n$, partition the set $\{1,2, \ldots, n\}$, when $n$ is odd. However, this does not hold when $n$ is even.

\section{Super edge-magic labelings of 2-Regular graphs union $K_{2}$}

The construction shown in section 2.1 can be slightly modified to give super edge-magic labelings of the union of $K_{2}$ and 2-regular graphs, we feel that this is interesting since Wallis proposes in [20] the following open question that appears in his book as research problem 2.15 .

Question 3.1. [20] For which values of $n$ is $C_{n} \cup K_{2}$ edge-magic?.

Using this technique we are able to find values of $n$ for which $C_{n} \cup K_{2}$ is super edge-magic, and therefore edge-magic.

Let $B_{m}=\left(b_{i j}\right)$ be the square matrix of dimension $m$ defined by $b_{i j}=1$ if either $i \leq m / 2$ and $j \equiv-2 i+2(\bmod m)$ or $m>i>m / 2$ and $j \equiv-2 i+1(\bmod m)$ and, $b_{i j}=0$, otherwise. Let $H_{m}$ be the digraph with vertex set $V\left(H_{m}\right)=\{1,2, \ldots, m\}$ and adjacency matrix $B_{m}$. Denote by $D_{m}=\left(d_{i j}\right)$ the square matrix of dimension $m$ defined by $d_{i j}=1$ if $j \equiv-2 i(\bmod m+1)$ and $d_{i j}=0$ otherwise.

Lemma 3.1. Let $m$ be an even integer. Then the digraph $H_{m}$ is isomorphic to the union of one arc together with a 1-regular digraph $J_{m-2}$, where $V\left(J_{m-2}\right)=\{i\}_{i=1}^{m-2}$ and $A\left(J_{m-2}\right)=D_{m-2}$. Furthermore, the digraph $H_{m}$ is super edge-magic.

Proof. By definition, it is clear that $(1, m) \in E\left(H_{m}\right)$. Moreover, an easy check shows that the function $f: V\left(J_{m-2}\right) \rightarrow V\left(H_{m}\right)$ defined by $f(x)=x+1$ is an isomorphism of $J_{m-2}$ and of the subdigraph of $H_{m}$ induced by $\{2,3, \ldots, m-1\}$.

Lemma 3.2. Let $n$ be an even integer. The digraph $J_{n}$ contains a cycle of length $k$ if and only if there exists $x \in\{1,2, \ldots, n\}$ such that $k$ is the minimum $i$ with

$$
\left((-2)^{i}-1\right) x \equiv 0(\bmod n+1) \text {. }
$$


Proof. Let $x \in\{1,2, \ldots, n\}$ and consider the sequence $x_{0}, x_{1}, \ldots, x_{k}$, where $x_{0}=x$ and $x_{j} \equiv-2 x_{j-1}(\bmod n+1)$, for $j=1,2, \ldots, k$. Thus, we obtain that $x_{j} \equiv$ $(-2)^{j} x(\bmod n+1)$. By construction, $x$ is in a cycle of length $k$ if and only if $x_{j} \neq x$, for $j=1,2, \ldots, k-1$ and $x_{k}=x$. Therefore, $x$ is in a cycle of length $k$ if and only if $k$ is the minimum $i$ such that $\left((-2)^{i}-1\right) x \equiv 0(\bmod n+1)$.

Proposition 3.3. Let $n$ be an even integer such that $n+1$ is prime. Let $k$ be the minimum $i$ such that $(-2)^{i} \equiv 1(\bmod n+1)$. Then,

$$
\operatorname{und}\left(H_{n}\right) \cong \frac{n-1}{k} C_{k} \cup K_{2} .
$$

Proof. Let $k$ be the minimum $i$ such that $(-2)^{i} \equiv 1(\bmod n+1)$. By Lemma 3.2, we know that und $\left(J_{n}\right)$ contains a component isomorphic to a cycle of order $k$ as a subgraph. Furthermore, 1 is in the vertex set of this component. Suppose that $x \in\{1,2, \ldots, n\}$ belongs to a component of und $\left(J_{n}\right)$ isomorphic to a cycle of order $r$, then $r$ is the minimum $i$ such that $\left((-2)^{i}-1\right) x \equiv 0(\bmod n+1)$. Thus, since $n+1$ is prime, we have that $r$ is the minimum $i$ such that $(-2)^{i}-1 \equiv 0(\bmod n+1)$. That is, by hypothesis $r=k$.

Corollary 3.4. Let $n+1$ be a prime number. Then, $n$ is a dual shuffle prime if and only if, und $\left(H_{n+1}\right) \cong C_{n} \cup K_{2}$.

Proof. By Theorem 2.11 in [3], $n$ is a dual shuffle prime if and only if, -2 generates the multiplicative group $\mathbb{Z}_{n+1}^{*}$. Thus, by Proposition 3.3, $n$ is a dual shuffle prime if and only if, und $\left(H_{n+1}\right) \cong C_{n} \cup K_{2}$.

Lemma 3.5. Let $n$ be an even integer. If $n \equiv 0(\bmod 4)$ then $C_{n} \cup K_{2}$ is super edge-magic.

Proof. Suppose that $n \equiv 0(\bmod 4)$. Let $V\left(C_{n} \cup K_{2}\right)=\left\{v_{i}\right\}_{i=1}^{n} \cup\{x, y\}$ and $E\left(C_{n} \cup K_{2}\right)=\left\{v_{i} v_{i+1}\right\}_{i=1}^{n-1} \cup\left\{v_{n} v_{1}, x y\right\}$. Consider the labeling $f: V\left(C_{n} \cup K_{2}\right) \rightarrow$ $\{i\}_{i=1}^{n+2}$ defined by, $f(x)=1, f(y)=n+2$ and:

$$
f(v)= \begin{cases}i+1, & \text { if } v=v_{2 i-1} \text { and } 1 \leq i \leq n / 4, \\ i+1, & \text { if } v=v_{2 i} \text { and } n / 4+1 \leq i \leq n / 2, \\ i+1+n / 2 & \text { if } v=v_{2 i} \text { and } 1 \leq i \leq n / 4 \\ i+1+n / 2 & \text { if } v=v_{2 i-1} \text { and } n / 4+1 \leq i \leq n / 2\end{cases}
$$

Then, $f$ is a super edge-magic labeling of $C_{n} \cup K_{2}$.

The next corollary is an easy consequence of the previous lemma together with Corollary 3.4 and Lemma 3.1.

Corollary 3.6. Let $n$ be an integer. If $n \equiv 0(\bmod 4)$ or $n$ is a dual shuffle prime then $C_{n} \cup K_{2}$ is super edge-magic.

It is worth mentioning, that Park et al. gave a super edge-magic labeling of $C_{n} \cup K_{2}$, for $n$ even, $n \neq 10$, in [18]. However, their description was somewhat more complicated. Furthermore, the smallest and the largest labels in our labelings are assigned to the vertices of $K_{2}$. Next, we introduce a result that is, in fact, easily deduced from Lemma 3.2.

Let $n$ be an even integer and let $\Phi_{k} \subset\{1,2, \ldots, n\}$ such that $x \in \Phi_{k}$ if $k$ is the minimum $i$ with the property that $\left((-2)^{i}-1\right) x \equiv 0(\bmod n+1)$. 
Theorem 3.7. Let $n$ be an even integer. Then,

$$
J_{n} \cong \cup_{k=1}^{n} \frac{\left|\Phi_{k}\right|}{k} C_{k}
$$

Finally, we have the following result that can be obtained from Theorem 3.7 and Lemma 3.1.

Theorem 3.8. Let $n$ be an even integer. Then, the graph $K_{2} \cup\left(\cup_{k=1}^{n}\left(\left|\Phi_{k}\right| / k\right) C_{k}\right)$ is super edge-magic.

Notice that, by Theorem 3.7, we immediately obtain that the sets $\Phi_{k}$, for $1 \leq$ $k \leq n$, partition the set $\{1,2, \ldots, n\}$, when $n$ is even. However, this does not hold when $n$ is odd. The following open question is motivated by Question 1.1 and by the work conducted in this section.

Question 3.2. Characterize the (super) edge-magic graphs of the form $G \cup K_{2}$ for some 2-regular graph $G$.

\section{The edge-Magicness of $C_{n} * u C_{m}$}

Denote by $C_{m}^{u}$ the cycle $C_{m}$ with exactly one loop attached to some of its vertices $u$, that is $C_{m}^{u} \cong C_{1} * u C_{m}$. The main goal of this section is to state and prove the following result.

Theorem 4.1. Let $n \geq 3$ be odd and let $m \in \mathbb{N} \backslash\{1,2\}$. Assume that either $m$ is even or, $m$ is odd and $m \geq n$. If $C_{m}^{u}$ is (super) edge-magic, $u \in V\left(C_{m}\right)$, then the graph $C_{n} * u C_{m}$ is (super) edge-magic.

In order to prove this theorem, first of all we will state and prove the following two lemmas.

Lemma 4.2. Let $m$ be an even integer and let $D$ be the oriented graph obtained from $C_{m}^{u}$ induced by an orientation of $C_{m}$ in which the arrows go from one stable set of $C_{m}$ to the other one. Then there is a function $h: E(D) \rightarrow\left\{C_{n}^{+}, C_{n}^{-}\right\}$such that $\operatorname{und}\left(D \otimes_{h}\left\{C_{n}^{+}, C_{n}^{-}\right\}\right) \cong C_{n} * u C_{m}$.

Proof. Let $\vec{C}_{m}^{b}$ be an orientation of $C_{m}$ in which the arrows go from one stable set of $C_{m}$ to the other one, when $m$ is even. Notice that, by the definition of $*$, it is clear that $E(D)=E\left(C_{1}^{+}\right) \cup E\left(\vec{C}_{m}^{b}\right)$, where $D \cong C_{1}^{+} * u \vec{C}_{m}^{b}, u \in V\left(C_{m}\right)$. Moreover, by the definition of the $\otimes_{h}$-product,

$$
E\left(D \otimes_{h}\left\{C_{n}^{+}, C_{n}^{-}\right\}\right)=E\left(C_{1}^{+} \otimes_{h_{\mid E\left(C_{1}^{+}\right)}}\left\{C_{n}^{+}, C_{n}^{-}\right\}\right) \cup E\left(\vec{C}_{m}^{b} \otimes_{h_{\mid E\left(\vec{C}_{m}^{b}\right)}}\left\{C_{n}^{+}, C_{n}^{-}\right\}\right)
$$

and $C_{1}^{+} \otimes_{h_{\mid E\left(C_{1}^{+}\right)}}\left\{C_{n}^{+}, C_{n}^{-}\right\} \cong h\left(E\left(C_{1}^{+}\right)\right)$. Hence, we only have to find a function $h_{1}: E\left(\vec{C}_{m}^{b}\right) \rightarrow\left\{C_{n}^{+}, C_{n}^{-}\right\}$such that und $\left(\vec{C}_{m}^{b} \otimes_{h_{1}}\left\{C_{n}^{+}, C_{n}^{-}\right\}\right) \cong n C_{m}$. By Theorem 1.7, the graph induced by the product is und $\left(\vec{C}_{m}^{b} \otimes\left\{C_{n}^{+}\right\}\right) \cong n C_{m}$ and, each copy of $C_{m}$ contains a vertex of the form $(u, i)$, for $i \in\{1,2, \ldots, n\}$. Therefore, if we let $h_{1}$ be the constant function defined by $h_{1}(e)=C_{n}^{+}$, for each $e \in E\left(\vec{C}_{m}^{b}\right)$ the result follows.

Lemma 4.3. Let $m$ and $n$ be odd integers with $m \geq n$. Let $\vec{C}_{m}^{u}$ be the orientation of $C_{m}^{u}$ induced by a strong orientation of the cycle $C_{m}$. Then there is a function $h: E\left(\vec{C}_{m}^{u}\right) \rightarrow\left\{C_{n}^{+}, C_{n}^{-}\right\}$such that und $\left(\vec{C}_{m}^{u} \otimes_{h}\left\{C_{n}^{+}, C_{n}^{-}\right\}\right) \cong C_{n} * u C_{m}$. 
Proof. As in the previous proof, we only need to find a function $h_{1}: E\left(C_{m}^{+}\right) \rightarrow$ $\left\{C_{n}^{+}, C_{n}^{-}\right\}$such that und $\left(C_{m}^{+} \otimes_{h_{1}}\left\{C_{n}^{+}, C_{n}^{-}\right\}\right) \cong n C_{m}$. Assume that $m \neq n$, otherwise the result holds by Theorem 1.3. Since $n$ is odd, we have that the congruence relation

$$
m-2 r \equiv 0(\bmod n)
$$

can be solved for some $r$ such that $0<r<m$. Therefore, inheriting the notation of Theorem 1.9, by considering any function $h_{1}$ with $N_{2}\left(h_{1}^{-}\right)=r$, we have that Theorem 1.9 implies that und $\left(C_{m}^{+} \otimes_{h_{1}}\left\{C_{n}^{+}, C_{n}^{-}\right\}\right) \cong n C_{m}$.

Now, we are ready to prove Theorem 4.1.

Proof of Theorem 4.1. If $m \equiv 3(\bmod 4)$ and $m \geq n$, by Theorem 1.5, we know that $C_{n} * u C_{m}, u \in V\left(C_{m}\right)$ is super edge-magic, and hence edge-magic. In fact, using Theorem 1.2, Lemma 4.3 and the hypothesis on $C_{m}^{u}$ we obtain that $C_{n} * u C_{m}$ is (super) edge-magic for each odd integer $m$, with $m \geq n$. Thus, we only need to concentrate on the case when $m$ is even. This case follows from Theorem 1.2 and Lemma 4.2.

Consider the edge-magic labelings of $C_{4}^{u}$ and $C_{6}^{u}$ that appear in Figure 1. Hence, we immediately obtain the following corollary of Theorem 4.1.

Corollary 4.4. Let $n$ be an odd integer. Then,

(i) $C_{n} * u C_{4}$ is edge-magic, $u \in V\left(C_{4}\right)$.

(ii) $C_{n} * u C_{6}$ is edge-magic, $u \in V\left(C_{6}\right)$.
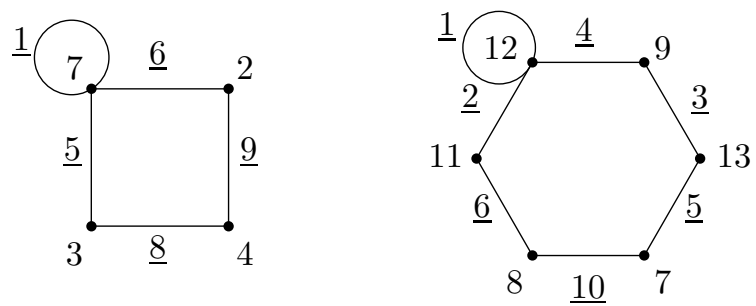

Figure 1. Edge-magic labelings of $C_{4}^{u}$ and $C_{6}^{u}$.

\section{The EDGe-MAgiCness of $C_{n} * u P_{m}$}

Let the graph $P_{m}^{l}$ be the path of order $m$ with a loop attached to one of the leaves, $l$, of $P_{m}$. Then we introduce the following lemma.

Lemma 5.1. The graph $P_{m}^{l}$ is edge-magic for every $m \in \mathbb{N}$, where $l$ is a leaf of $P_{m}$.

Proof. We will consider two cases, depending on the parity of $m$. Let $V\left(P_{m}^{l}\right)=$ $\left\{v_{i}\right\}_{i=1}^{m}$ and $E\left(P_{m}^{l}\right)=\left\{v_{i} v_{i+1}\right\}_{i=1}^{m-1} \cup\left\{v_{m} v_{m}\right\}$. 


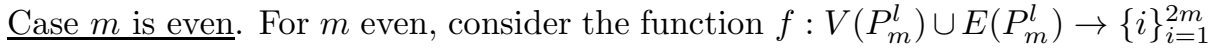
defined by the rule:

$$
\begin{aligned}
f\left(v_{i}\right) & = \begin{cases}(i+1) / 2 & \text { if } i \text { is odd, } \\
(i+m+2) / 2 & \text { if } i \text { is even, }\end{cases} \\
f\left(v_{i} v_{j}\right) & =\frac{5 m+6}{2}-f\left(v_{i}\right)-f\left(v_{j}\right), v_{i} v_{j} \in E\left(P_{m}^{l}\right) .
\end{aligned}
$$

Then $f$ is an edge-magic labeling of $P_{m}^{l}$.

Case $m$ is odd. For $m$ odd, consider the function $f: V\left(P_{m}^{l}\right) \cup E\left(P_{m}^{l}\right) \rightarrow\{i\}_{i=1}^{2 m}$ defined by the rule:

$$
\begin{aligned}
f\left(v_{i}\right) & = \begin{cases}(i+m+2) / 2 & \text { if } i \text { is odd, } \\
i / 2 & \text { if } i \text { is even, }\end{cases} \\
f\left(v_{i} v_{j}\right) & =\frac{5 m+5}{2}-f\left(v_{i}\right)-f\left(v_{j}\right), v_{i} v_{j} \in E\left(P_{m}^{l}\right) .
\end{aligned}
$$

Then $f$ is an edge-magic labeling of $P_{m}^{l}$.

At this point, we are ready to state and prove the following theorem.

Theorem 5.2. Let $n \geq 3$ be an odd integer and consider the cycle $C_{n}$. Also consider any path $P_{m}$ and let $l$ be any leaf of $P_{m}$. Then the graph $C_{n} * l P_{m}$ is edge-magic.

Proof. Let $\vec{P}_{m}$ be an orientation of $P_{m}$ that allows us to travel from one leaf of $P_{m}$ to the other one following the sense of the arrows. Let $\Gamma=\left\{C_{n}^{+}, C_{n}^{-}\right\}$and consider any function $h: E\left(\vec{P}_{m}^{l}\right) \rightarrow \Gamma$, where $\vec{P}_{m}^{l}$ is the digraph obtained from $\vec{P}_{m}$ by attaching a loop at vertex $l$. Clearly, $E\left(\vec{P}_{m}^{l}\right)=E\left(\vec{P}_{m}\right) \cup\{(l, l)\}$ and, by definition of the $\otimes_{h}$-product,

$$
E\left(\vec{P}_{m}^{l} \otimes_{h} \Gamma\right)=E\left(\vec{P}_{m} \otimes_{h_{\mid E\left(\vec{P}_{m}\right)}} \Gamma\right) \cup E\left(\{(l, l)\} \otimes_{h_{\mid\{(l, l)\}}} \Gamma\right) .
$$

Thus, by Theorem 1.8 we have that und $\left(\vec{P}_{m} \otimes_{h_{\mid E(\vec{P} m)}} \Gamma\right) \cong n P_{m}$ and, since each copy of $P_{m}$ contains a vertex of the form $(l, i)$, for $i \in\{1,2, \ldots, n\}$, we get

$$
\operatorname{und}\left(\vec{P}_{m}^{l} \otimes_{h} \Gamma\right) \cong C_{n} * l P_{m} .
$$

Since both elements of $\Gamma$ are super edge-magic and, by Lemma $5.1, \vec{P}_{m}^{l}$ is edgemagic for every $n \in \mathbb{N}$, Theorem 1.2 implies that $C_{n} * l P_{m}$ is edge-magic.

Lemma 5.3. The graph $P_{m}^{l}$ is super edge-magic for every $m \in \mathbb{N}$, with $m \equiv 2$ $(\bmod 4)$.

Proof. Let $V\left(P_{m}^{l}\right)=\left\{v_{i}\right\}_{i=1}^{m}$ and $E\left(P_{m}^{l}\right)=\left\{v_{i} v_{i+1}\right\}_{i=1}^{m-1} \cup\left\{v_{m} v_{m}\right\}$. The result is trivial for $P_{2}^{l}$. Assume that $m \geq 6$ and consider $k \in \mathbb{N}$ with $m=2(2 k+1), k \geq 1$. Let $f: V\left(P_{m}^{l}\right) \rightarrow\{i\}_{i=1}^{m}$ be defined by

$$
f\left(v_{i}\right)= \begin{cases}k-j+2, & \text { if } i=2 j-1 \quad j=1, \ldots, k+1, \\ 3 k-j+3, & \text { if } i=2 j-1 j=k+2, \ldots, 2 k+1, \\ m, & \text { if } i=2 k+2, \\ 3 k-j+2, & \text { if } i=2 j \quad j=1, \ldots, k, \\ 5 k-j+3, & \text { if } i=2 j \quad j=k+2, \ldots, 2 k+1 .\end{cases}
$$

Then $f$ is a super edge-magic labeling of $P_{m}^{l}$. 
Corollary 5.4. Let $n \geq 3$ be an odd integer and let $m \equiv 2(\bmod 4)$. Then the graph $C_{n} * l P_{m}$ is super edge-magic, where $l$ is any leaf of $P_{m}$.

Lemma 5.3 suggests the following open question.

Question 5.1. For which values of $m$ is the graph $P_{m}^{l}$ super edge-magic?

Let $P_{m}^{l, s}$ be the path of order $m$ with a loop attached to each of the leaves, $l$ and $s$, of $P_{m}$. Then we introduce the following lemma.

Lemma 5.5. The graph $P_{m}^{l, s}$ is super edge-magic for every even $m \in \mathbb{N}$, where $l$ and $s$ are the leaves of $P_{m}$.

Proof. Let $V\left(P_{m}^{l, s}\right)=\left\{v_{i}\right\}_{i=1}^{m}$ and $E\left(P_{m}^{l, s}\right)=\left\{v_{i} v_{i+1}\right\}_{i=1}^{m-1} \cup\left\{v_{1} v_{1}\right\} \cup\left\{v_{m} v_{m}\right\}$. The result is trivial for $P_{2}^{l, s}$. For $m=4$ and $m=8$ the sequences $2-1-4-3$ and $3-4-1-7-2-8-5-6$, define a super edge-magic labeling of $P_{4}^{l, s}$ and $P_{8}^{l, s}$, respectively. For the remaining values of $m$, we will consider two cases.

Case $m=4 k(k>2)$. We consider the function $f: V\left(P_{m}^{l, s}\right) \rightarrow\{i\}_{i=1}^{m}$ defined by the rule:

$$
f\left(v_{i}\right)= \begin{cases}k+1, & \text { if } i=1, \\ k+1+j, & \text { if } i=2 j, \quad j=1, \ldots, k-1, \\ 3 k+j, & \text { if } i=2 j+1, \quad j=1, \ldots, k-2, \\ 1, & \text { if } i=2 k-1, \\ 4 k-1, & \text { if } i=2 k, \\ 4 k+1-f\left(v_{2 k+1-j}\right), & \text { if } i=2 k+j, \quad j=1, \ldots, 2 k .\end{cases}
$$

Then $f$ is a super edge-magic labeling of $P_{m}^{l, s}$.

Case $m=4 k+2(k \geq 1)$. We consider the function $f: V\left(P_{m}^{l, s}\right) \rightarrow\{i\}_{i=1}^{m}$ defined by the rule:

$$
f\left(v_{i}\right)= \begin{cases}k+2-j, & \text { if } i=2 j-1, \quad j=1, \ldots, k+1, \\ 3 k+2-j, & \text { if } i=2 j, \quad j=1, \ldots, k, \\ 4 k+3-f\left(v_{2 k+3-j}\right), & \text { if } i=2 k+j, \quad j=2, \ldots, 2 k+2 .\end{cases}
$$

Then $f$ is a super edge-magic labeling of $P_{m}^{l, s}$.

Notice that the graph und $\left(\vec{P}_{m}^{l, s} \otimes C_{n}^{+}\right)$is isomorphic to the graph obtained from $P_{2} \times C_{n}$ by replacing each edge joining two vertices of the different copies of $C_{n}$ by a path of order $m$. By Theorem 1.2 and Lemma 5.5 we obtain the next result.

Corollary 5.6. Let $m$ be an even integer and $n$ be odd. Then, the graph und( $\left.\vec{P}_{m}^{l, s} \otimes C_{n}^{+}\right)$is super edge-magic.

\section{Further RESUlts AND CONClusions}

Using similar techniques as the ones presented in sections 4 and 5, we can extend the family of super edge-magic graphs. In particular we can extend the family of 2 -regular super edge-magic graphs. For instance, when $m$ is a dual shuffle prime we can obtain the following result, which is a particular case of a more general result found in [17].

Theorem 6.1. Let $m$ be a dual shuffle prime and let $n$ be an odd integer $n \geq 3$. Then $C_{m n} \cup C_{n}$ is super edge-magic. 
Proof. Lemma 2.1 and Corollary 2.8 imply that $C_{m} \cup C_{1}$ is super edge-magic. Thus, by Theorem 1.2, for any function $h: E\left(C_{n}^{+}\right) \rightarrow\left\{C_{m}^{+} \cup C_{1}^{+}, C_{m}^{-} \cup C_{1}^{+}\right\}$, the graph und $\left(C_{n}^{+} \otimes_{h}\left\{C_{m}^{+} \cup C_{1}^{+}, C_{m}^{-} \cup C_{1}^{+}\right\}\right)$is super edge-magic. Moreover, by definition of the $\otimes_{h}$-product,

$$
C_{n}^{+} \otimes_{h}\left\{C_{m}^{+} \cup C_{1}^{+}, C_{m}^{-} \cup C_{1}^{+}\right\} \cong\left(C_{n}^{+} \otimes_{h^{\prime}}\left\{C_{m}^{+}, C_{m}^{-}\right\}\right) \cup C_{n}^{+},
$$

for some function $h^{\prime}: E\left(C_{n}^{+}\right) \rightarrow\left\{C_{m}^{+}, C_{m}^{-}\right\}$. Thus, we only have to find a function $h^{\prime}: E\left(C_{n}^{+}\right) \rightarrow\left\{C_{m}^{+}, C_{m}^{-}\right\}$such that und $\left(C_{n}^{+} \otimes_{h^{\prime}}\left\{C_{m}^{+}, C_{m}^{-}\right\}\right) \cong C_{m n}$. Since $n$ is odd, we have that $r=(n-1) / 2$ satisfies the congruence relation $n-2 r \equiv 1(\bmod m)$. Hence, inheriting the notation of Theorem 1.9, by considering any function $h^{\prime}$ with $N_{2}\left(h^{\prime-}\right)=r$, we have that Theorem 1.9 implies that und $\left(C_{n}^{+} \otimes_{h^{\prime}}\left\{C_{m}^{+}, C_{m}^{-}\right\}\right) \cong C_{m n}$. Therefore, the result follows.

Similarly, by Theorem 1.3 or Theorem 1.9 and the fact that $C_{m} \cup K_{2}$ is super edge-magic [18], for $m$ even and $m \neq 10$, we obtain the next result

Theorem 6.2. Let $m \geq 3$ and $n \geq 4$ be integers with $m$ even, $m \neq 10$, $n$ odd and either $\operatorname{gcd}(m, n)=1$ or $m>n$. Then $C_{m n} \cup n K_{2}$ is super edge-magic.

Proof. Lemma 3.1 and Corollary 3.6 imply that $C_{m} \cup K_{2}$ is super edge-magic. Thus, by Theorem 1.2, for any function $h: E\left(C_{m}^{+} \cup \vec{K}_{2}\right) \rightarrow\left\{C_{n}^{+}, C_{n}^{-}\right\}$, the graph $\operatorname{und}\left(\left(C_{m}^{+} \cup \vec{K}_{2}\right) \otimes_{h}\left\{C_{n}^{+}, C_{n}^{-}\right\}\right)$is super edge-magic. Moreover, by definition of the $\otimes_{h}$-product,

$$
\left(C_{m}^{+} \cup \vec{K}_{2}\right) \otimes_{h}\left\{C_{n}^{+}, C_{n}^{-}\right\} \cong\left(C_{m}^{+} \otimes_{h_{\mid E\left(C_{m}^{+}\right)}}\left\{C_{n}^{+}, C_{n}^{-}\right\}\right) \cup\left(\vec{K}_{2} \otimes_{h_{\mid E\left(\vec{K}_{2}\right)}}\left\{C_{n}^{+}, C_{n}^{-}\right\}\right),
$$

and by Theorem 1.8, und $\left(\vec{K}_{2} \otimes_{h_{\mid E\left(\vec{K}_{2}\right)}}\left\{C_{n}^{+}, C_{n}^{-}\right\}\right) \cong n K_{2}$. Thus, we only have to find a function $h_{1}: E\left(C_{m}^{+}\right) \rightarrow\left\{C_{n}^{+}, C_{n}^{-}\right\}$such that und $\left(C_{m}^{+} \otimes_{h_{1}}\left\{C_{n}^{+}, C_{n}^{-}\right\}\right) \cong C_{m n}$. If $\operatorname{gcd}(m, n)=1$ then, by Theorem 1.3, any constant function $h_{1}$ satisfies this property. Suppose now that $m>n$. Since $m$ is even and $n$ is odd, we have that the congruence relation $m-2 r \equiv 1(\bmod n)$ can be solved with $0<r<m$. Hence, inheriting the notation of Theorem 1.9, by considering any function $h_{1}$ with $N_{2}\left(h_{1}^{-}\right)=r$, we have that Theorem 1.9 implies that und $\left(C_{m}^{+} \otimes_{h_{1}}\left\{C_{n}^{+}, C_{n}^{-}\right\}\right) \cong C_{m n}$. Therefore, the result follows.

Notice that, since the super edge-magic labeling of $C_{n} \cup K_{2}$ obtained in section 3 assignes the smallest amb the largest labels to the vertices of $K_{2}$, by removing the labels of $K_{2}$ and shifting the labels of $C_{n}$ by one unit down, we can obtain a labeling of $C_{n}$ with one chord (which is not necessarily unique). In particular, we can obtain the next results.

Lemma 6.3. Let $m$ be an even integer. If $m$ is a shuffle prime then the cycle $C_{m}$ with exactly one chord joining antipodal vertices is super edge-magic. Moreover, it admits at least $m / 2$ nonisomorphic super edge-magic labelings.

Proof. Consider the labeling of $C_{m} \cup K_{2}$ introduced in section 3, recall that the smallest and the largest labels are assigned to the vertices of $K_{2}$. An easy check shows that the sum $f(a)+f(b)$, where $a, b \in V\left(K_{2}\right)$ is the same as the sum $f(u)+$ $f(v)$, for any pair of antipodal vertices of the cycle $C_{m}$. Thus, by removing the labels of $K_{2}$ and shifting the labels of $C_{n}$ by one unit down, we can obtain a labeling of $C_{n}$ with one chord, by joining any pair of antipodal vertices.

Using a similar idea to the one used in the proof of the previous lemma, the next result can be easily proved from the labeling of $C_{m} \cup K_{2}$ introduced in Lemma 3.5. 
Lemma 6.4. Let $m$ be an integer with $m \equiv 0(\bmod 4)$. Then the cycle $C_{m}$ with exactly one chord joining two vertices at distance $2+4 i$, for some $i=0,1, \ldots,\lceil\mathrm{m} / 8\rceil$, is super edge-magic.

Similarly, it can be checked that, in Theorem 6.2, the labeling of $C_{m n} \cup m K_{2}$ induced by the product [11] (a vertex $(u, i)$ receives the label $n(f(u)-1)+i$, where $f$ is the labeling of $C_{m} \cup K_{2}$ introduced in section 3 and each vertex of $C_{n}$ is identified with its label in a given super edge-magic labeling), assignes the smallest amb the largest labels to the vertices of $m K_{2}$. Hence, by shifting the labels of the cycle $m$ units down, we can obtain a super edge-magic labeling of a cicle $C_{m}$ together with $m$ chords (which is not necessarily unique). Figures 2 and 3 show an example of these constructions.

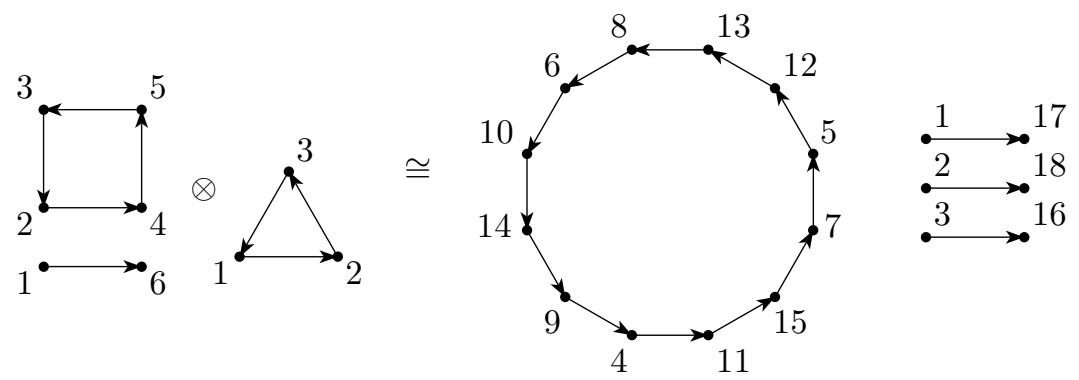

Figure 2. A super edge-magic labeling of $C_{12}^{+} \cup 3 \vec{K}_{2}$ induced by the product $\left(C_{4}^{+} \cup \vec{K}_{2}\right) \otimes C_{3}^{+}$.

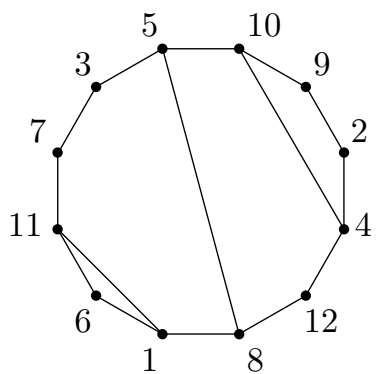

Figure 3. A super edge-magic labeling of $C_{12}$ with three chords obtained from the labeling of Figure 2.

It is worth the while mentioning that Bača and Milller paid special attention to super edge-magic labelings of cycles with chords, in [4]. In this paper we have obtained some of their results, but by using absolutely different techniques, that seem to be very promising for future work. The interested reader can consult this source for further information on this problem. 
6.1. Conclusions. In this paper we have used operations on strings in order to obtain super edge-magic labelings of 2-regular graphs (Theorem 2.10), and of 2regular graphs union $K_{2}$ (Theorem 3.8). Our point of view has singled out two interesting relations with sequences of integers, one of such relations being with dual shuffle primes (Corollary 2.8 and Corollary 3.4) and the other one being with the Jacobsthal sequence (Corollary 2.5 and Theorem 2.9). By using the $\otimes_{h}$-product of digraphs, we have also studied the edge-magicness of $C_{n} * u C_{m}, u \in V\left(C_{m}\right.$ (Theorem 4.1) and of $C_{n} * l P_{m}, l$ a leaf of $P_{m}$ (Theorem 5.2). Moreover, the combination of the $\otimes_{h}$-product together with the labelings introduced in sections 2 and 3 have allowed us to present results on the edge-magicness of $C_{m} \cup n K_{2}$ (Theorem 6.2) and of cycles with chords.

Acknowledgements The authors want to thank the anonymous referees for their careful reading of the paper and the suggestions they made, which helped to increase the quality of the paper.

The research conducted in this document by the first and third authors has been supported by the Spanish Research Council under project MTM2011-28800-C02-01 and by the Catalan Research Council under grant 2009SGR1387.

\section{REFERENCES}

[1] B. D. Acharya and S. M. Hegde, Strongly indexable graphs, Discrete Math. 93 (1991) $123-129$.

[2] A. Ahmad, F. A. Muntaner-Batle, M. Rius-Font, On the product $C_{m}^{+} \otimes_{h}\left\{C_{n}^{+}, C_{n}^{-}\right\}$and other related topics, Ars Combin. in press.

[3] P. R. J. Asveld, Permuting Operations on Strings and Their Relation to Prime Numbers, Discrete Appl. Math. 159 (17) (2011) 1915-1932.

[4] M. Bača and M. Miller, Super Edge-Antimagic Graphs, BrownWalker Press, Boca Raton, 2008.

[5] G. Chartrand and L. Lesniak, Graphs and Digraphs, second edition. Wadsworth \& Brooks/Cole Advanced Books and Software, Monterey (1986).

[6] H. Enomoto, A. Lladó, T. Nakamigawa and G. Ringel, Super edge-magic graphs, SUT J. Math. 34 (1998) 105-109.

[7] R. M. Figueroa-Centeno, R. Ichishima and F. A. Muntaner-Batle, The place of super edge-magic labelings among other classes of labelings, Discrete Math. 231 (1-3) (2001) 153-168.

[8] R. M. Figueroa-Centeno, R. Ichishima and F. A. Muntaner-Batle, Magical coronations of graphs, Australasian J. Combin. 26 (2002) 199-208.

[9] R. M. Figueroa-Centeno, R. Ichishima and F. A. Muntaner-Batle, On super edge-magic labelings of certain disjoint unions of graphs, Australasian J. Combin. 32 (2005) 225-242.

[10] R. M. Figueroa-Centeno, R. Ichishima, F. A. Muntaner-Batle and A. Oshima, A magical approach to some labeling conjectures, Discuss. Math. Graph Theory 311 (2011) 79-113.

[11] R. M. Figueroa-Centeno, R. Ichishima, F. A. Muntaner-Batle and M. Rius-Font, Labeling generating matrices, J. Comb. Math. Comb. Comput. 67 (2008) 189-216.

[12] J. Holden, D. McQuillan and J. M. McQuillan, A conjecture on strong magic labelings of 2-regular graphs, Discrete Math. 309 (2009) 4130-4136.

[13] R. Ichishima, S. C. López, F. A. Muntaner-Batle, M. Rius-Font, The power of digraph products applied to labelings, Discrete Math. 312 (2012) 221-228.

[14] J.A. Gallian, A dynamic survey of graph labeling, Electron. J. Combin. 14 (2011) $\sharp$ DS6

[15] A. Kotzig and A. Rosa, Magic valuations of finite graphs, Canad. Math. Bull. 13 (1970) 451-461.

[16] S. C. López, F. A. Muntaner-Batle, M. Rius-Font, Bi-magic and other generalizations of super edge-magic labelings, Bull. Austral. Math. Soc. 84 (2011) 137-152.

[17] S.C. López, F. A. Muntaner-Batle, M. Rius-Font, Labeling constructions using the $\otimes_{h^{-}}$ product, Discrete Appl. Math. (2013) http://dx.doi.org/10.1016/j.bbr.2011.03.031. 
[18] J. Y. Park, J. H. Choi, J.-H. Bae, On super edge-magic labeling of some graphs, Bull. Korean Math. Soc. 45 (1) (2008) 11-21.

[19] N.J.A. Sloane, On-Line Encyclopedia of Integer Sequences, http://oeis.org/Seis.html.

[20] W.D. Wallis, Magic graphs. Birkhaüser, Boston (2001).

Universitat Politècnica de Catalunya. Barcelonatech, Dept. Matemàtica Apl. IV; Esteve Terrades 5, 08860 Castelldefels, Spain

E-mail address: susana@ma4.upc.edu

Graph Theory and Applications Research Group. School of Electrical Engineering and Computer Science. Faculty of Engineering and Bullt Environment. The University of NewCastle. NSW 2308 Australia

E-mail address: famb1es@yahoo.es

Universitat Politècnica de Catalunya. Barcelonatech, Dept. Matemàtica Apl. IV; Esteve Terrades 5, 08860 Castelldefels, Spain

E-mail address: mriusfont@gmail.com 